\section{ON THE MYDRIATIC PROPERTIES OF HYDROBROMATE OF HYOSCINE.}

BY JOHN TWEEDY, F.R.CS.ENG.,

PROFESSOR OF OPHTHALMIC MEDICINE AND SURGERY IN UNIVERSITY COLLEGE, AND SURGEON TO THE ROYAL LONDON OPHTHALMIO HOSPITAL.

THE researches of Ladenburg have shown that three different mydriatic bodies are contained in the atropaceous alkaloids-namely, pure atropine in belladonna and stramonium; pure hyoscyamine (crystalline) in belladonna stramonium, hyoscyamus, and duboisia; and lastly, hyoscine, which is associated with hyoscyamine, in hyoscyamus. Pure duboisine is identical with pure hyoscyamine. The relationship which scopoline, another atropaceous mydriatic obtained from Scopolia japonica, bears, chemically and physiologically, to those just mentioned, has not, I believe, up to the present been precisely determined. Though atropine,

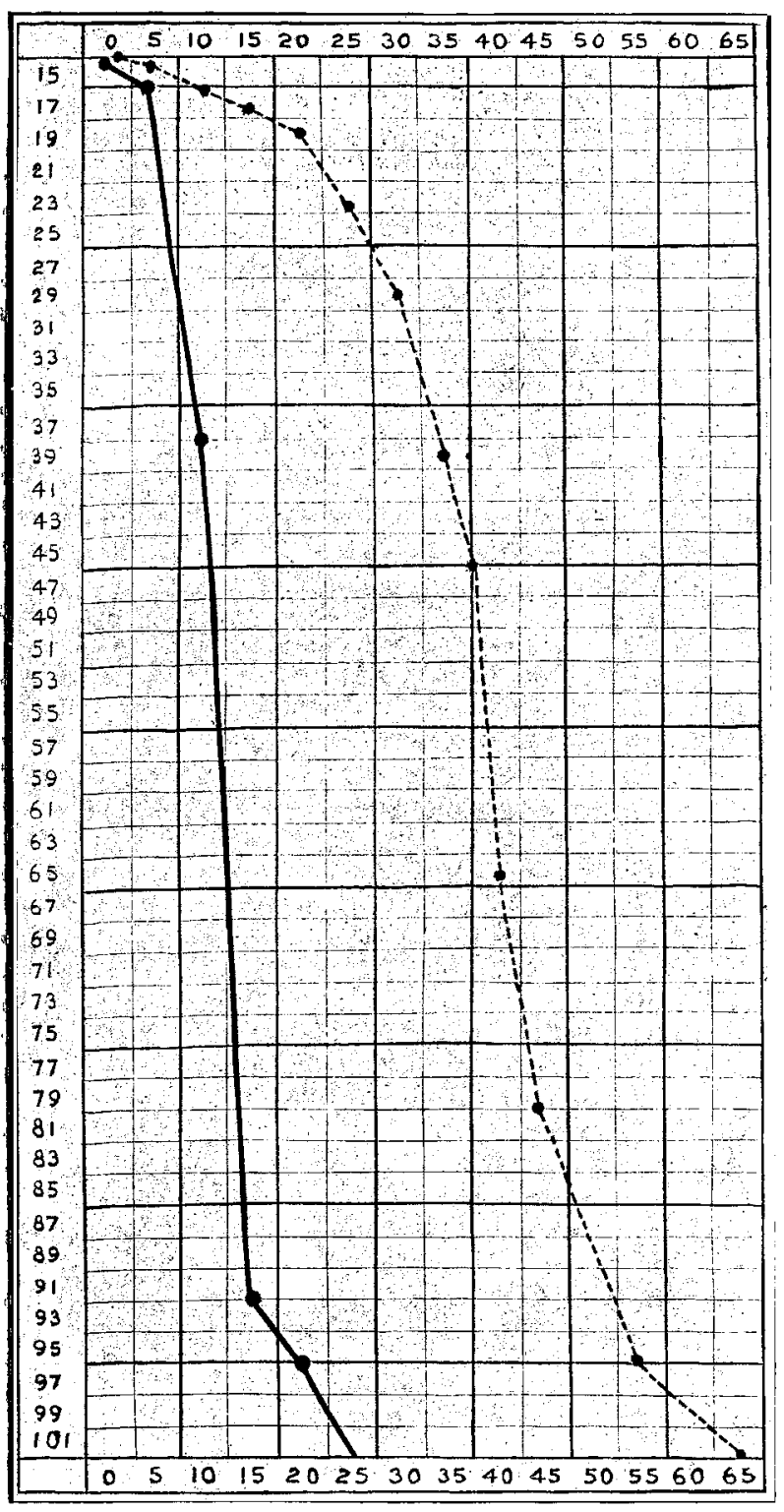

hyoscyamine, and hyoscine are isomeric, they possess somewhat different physical, chemical, and physiological properties. The last named, hyoscine, is the most recent of these therapeutical agents, and appears to have been little used in England. In America, on the other hand, it has been extensively employed, chiefly on the recommendation of the very elaborate experimental and clinical investigations of Professor H.C. Wood of Philadelphia. In the current (November) number of The Practitioner, Dr. Mitchell Bruce has called attention to the remarkable efficacy of hyoscine in controlling " those conditions of cerebral excitement variously known as delirium, mania, and insomnia, with restlessness." The prompt and satisfactory results which Dr. Mitchell Bruce had obtained suggested a special trial of hyoscine as a mydriatic.
In commerce there are three crystalline salts of hyoscine : the hydriodate, the hydrobromate, and the hydrochlorate. The first occurs in large prismatic orange-coloured crystals, which are not freely soluble in water; the hydrobromate in small crystals, which are readily soluble in water. Partly on this account, and partly because the hydriodate might contain free iodine, the hydrobromate seemed better suited to ophthalmic purposes. The experiments whose results are here recorded were made with a solution of the latter salt of the strength of 1 in 200, prepared by Mr. Martindale of New Cavendish-street. The solution proved to be a rapid, powerful, and unirritating dilator of the pupil alike in the healthy and in the inflamed iris, and a paralyser of the accommodation. Notwithstanding its great activity when given internally or subcutaneously, I have not yet met with an instance of its producing toxic effects such as occasionally follow the use of duboisine, though this contingency may be possiblewith strongersolutions. Having tester the general mydriatic action of the drug in healthy and in diseased states of the eye, I asked Mr. E. T. Collins, senior housesurgeon at the Moorfields Ophthalmic Hospital, to undertake some comparative observations with a solution of atropine of corresponding strength. The results of his observations are shown in the annexed diagram. The horizontal rows of figures indicate the time of action in minutes, the vertical column the position of the nearest point of distinct vision $(p p)$ in centimetres. The effect of the hyoscine is represented by the continuous line; that of atropine by the broken line.

Before applying the solutions, the "nearest point" was ascertained to be at 15 centimetres in both eyes. Hyoscine was applied to the right eye, and atropine to the left. Hyoscine removed the nearest point to 38 centimetres in ten minutes, whereas it took the atropine thirty-five minutes to do this. In twenty-five minutes the accolomodation was completely paralysed by hyoscine, the nearest point having receded to 100 centimetres; whereas with atropine this was not attained for sixty-five minutes. Similarly, the pupil was fully dilated by hyoscine in fifteen minutes, while it required twenty-five minutes to produce an equal effect. (See diagram.) Further, the effects of hyoscine also last longer than those of atropine, and are not so easily counteracted by eserine.

The indication seems to be that, if hyoscine is to have a place in ophthalmic therapeutics, it will be chiefly in cases of iritis and in other conditions where it is desirable to produce as much dilatation of the pupil as possible and for as long a time as possible. It is worthy of note that the application of byoscine to the eye does not seem to cause the unpleasant taste and feeling in the throat which often follows the use of atropine. This circumstance accords with the observations of Dr. Bruce and others, that when given internally it does not produce unpleasant dryness of the throat and skin like atropine.

Harley-street, $\mathrm{W}$.

\section{SOME CASES OF LOCAL NUMBNESS OF} THE EXTREMITIES,

WITH COMPARISONS BETWEEN LOCAL SYNCOPE AND "NIGHT PALSY."

BY J. EDWARD SQUIRE, M.D. LOND, M.R.C.P., PHYSICIAN TO THE NORTH LONDON HOSPITAL FOR DISEASES OF THE CHEST ; PHYSTCIAN TO ST. GEORGE'S AND ST. JAMES'S DISPENSARY.

CASE 1.-E. G—, aged eighteen, tailor, came under my care in March, 1884, complaining of numbness or deadness of the fingers and of giddiness. He is a tall, broad-built, well-developed lad, with thick lips, fair complexion, and light wavy hair. The numbness of his hands is not constant, but occurs about once every day, generally about midday. It appears to be induced by washing in cold water when the hands are already cold. Although he washes his hands in cold water when he gets up, this does not make them "go off," as, having just got out of bed, they are then warm." At several of his visits I had the opportunity of seeing his bands in the condition for the relief of which he applied, and the following was their appearance on one occasion: "The middle finger of the right hand is quite white to the centre joint; the rest of the hand, with the 